\title{
Time-saving fixed lingual retainer using DuraLay resin transfer
}

\author{
Shin-Jae Lee, DDS, MSD, PhD, ${ }^{a}$ Jong-An Ihm, DDS, MSD, ${ }^{b}$ and Sug-Joon Ahn, DDS, MSD, PhD \\ Seoul, South Korea
}

$\mathbf{T}$ There is general agreement on the necessity of fixed lingual retainers to prevent relapse in many patients after active orthodontic treatment. ${ }^{1-3}$ Several methods for delivering fixed lingual retainers have been introduced. ${ }^{2,4-9}$ However, bonding a lingual retainer is still challenging because it requires long working time and has a risk of contamination from saliva and moisture, which can cause bonding failure. In addition, it is difficult to adapt the retainer wire to perfectly fit the lingual surfaces of an anterior tooth. We developed a method of fabricating a fixed lingual retainer using DuraLay resin (Reliance Dental Manufacturing, Worth, Ill) and have used this method with satisfactory results for nearly 10 years on thousands of patients. This technique has proven to be a reliable, convenient, and time-saving procedure.

\section{PROCEDURE}

A few weeks before debonding, take an impression and pour an accurate cast in hard stone. Placing the fixed lingual retainer before debonding provides several advantages, including avoiding the relapse that might occur immediately after debonding, giving a patient time to adapt to the lingual retainer before debonding, and reducing complications at the debonding appointment.

Gently bend a length of multi-stranded wire to accurately fit the cast. Check the adaptation of the wire to ensure passive location against all tooth surfaces to be retained. Our preferred material is 0.0175 -in multistranded wire (Unitek Coaxial, 3M/Unitek, Monrovia, Calif). This 6-stranded wire has a relatively smooth

\footnotetext{
From the Department of Orthodontics, College of Dentistry, Seoul National University, Seoul, South Korea.

${ }^{\mathrm{a}}$ Assistant professor.

${ }^{\mathrm{b} C l i n i c a l}$ fellow.

cFull-time instructor.

Reprint requests to: Shin-Jae Lee, DDS, MSD, PhD, Assistant Professor, Department of Orthodontics, College of Dentistry, Seoul National University, 28-22 Yunkeun-Dong, Chongro-Ku, Seoul 110-744, South Korea; e-mail, nonext@empal.com.

Am J Orthod Dentofacial Orthop 2004;125:203-5

Submitted, December 2002; revised and accepted, March 2003. 0889-5406/\$30.00

Copyright $\odot 2004$ by the American Association of Orthodontists. doi:10.1016/j.ajodo.2003.03.004
}

surface conformation and offers increased mechanical retention and enough flexibility to allow physiologic movement of the teeth. ${ }^{10,11}$

Apply DuraLay separating medium to the cast and fix the shaped wire to the cast. Then mix the DuraLay resin and apply it gently between the distal surface of the second premolar and the mesial surface of the first molar (in case of 3-3 lingual retainer, between the contact points of the canine and premolar) (Fig 1). DuraLay resin is easy to manipulate and requires less time than other resin materials because it is fast setting and easy to detach from the cast. In addition, DuraLay resin is known to have good dimensional stability. ${ }^{12,13}$

Set Nola dry field system (Nola Specialties, Hilton Head Island, SC) in position and meticulously clean, etch, and prime the teeth to be bonded. Instead of a common air syringe, using warm, dry air at this stage is helpful. The warm, dry air is especially advantageous when we treat a patient with gingival recession and hypersensitive teeth.

Remove the wire from the cast and accurately transfer it to the teeth, using the DuraLay resin guide. Fix the transfer resin portion firmly with utility wax (Fig 2). Each tooth is then bonded individually with the Transbond XT system (3M/Unitek). ${ }^{14,15}$ First, bond all teeth other than the 2 terminal teeth. Cut the terminal portions of the wire and remove the DuraLay resin, and then bond the 2 terminal ends. The wire ends should not be exposed. Bond each resin portion carefully so that it tapers to a smooth, feathered edge and ensures accessibility by a toothbrush. At this stage, we manipulate the light-curing bonding resin with 2 blades of resincontouring instruments (GC 11, GC Co., Tokyo, Japan); this has proven useful in resin filling (Fig 3, A). Some minor polishing might be required to smooth the resin surface so that food impaction and tongue irritation are minimized. We use a Soflex (3M/Unitek) bur for this purpose (Fig 3, B). The finished retainer is shown in Figure 4.

\section{CONCLUSIONS}

DuraLay is a methylmethacrylate material widely used in fixed prosthodontics; it provides dimensional 


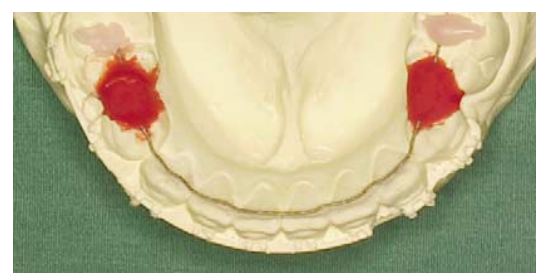

Fig 1. After length of multi-stranded wire is bent to fit cast, DuraLay resin is applied to hold wire between second premolar and mesial surface of first molar.

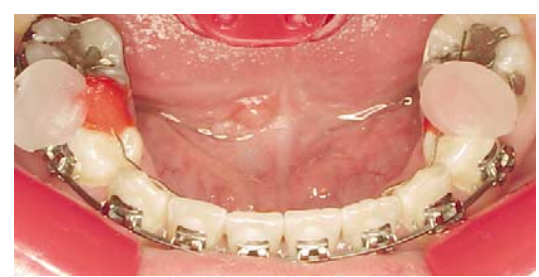

Fig 2. Transfer resin portion is firmly fixed with utility wax in mouth for 5-5 lingual retainer.
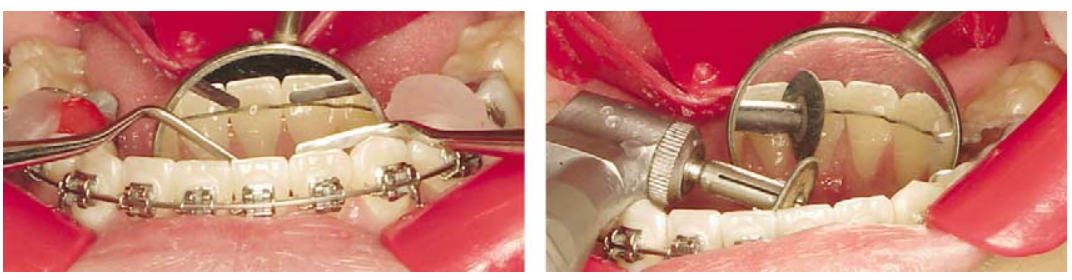

Fig 3. A, Each tooth is bonded individually with Transbond XT system; B, minor polishing is required to smooth resin surface.
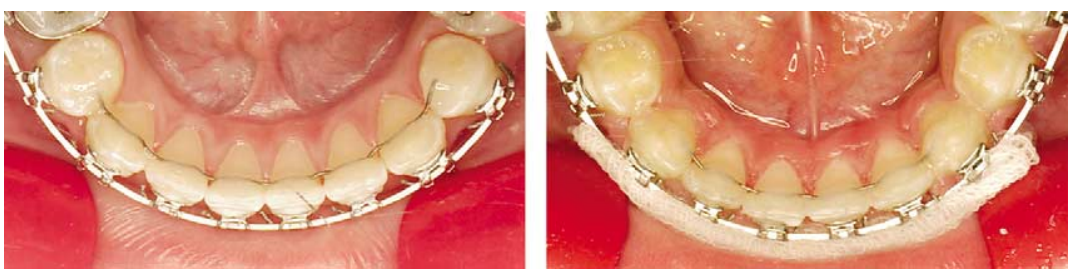

Fig 4. Finished lingual retainers. A, 5-5 fixed lingual retainer; B, 3-3 lingual retainer.

stability and convenience. As a transfer material, it seems to be appropriate in orthodontics, also. The technique described above is simple and accurate. Additionally, because the terminal portions of the retainer wire are firmly held in the mouth, the clinician can place the retainer alone, without fear of contamination or displacement of the wire. This procedure can offer comfortable manipulation, acceptable stabilization, and shorten both chair and laboratory times.

\section{REFERENCES}

1. Bearn DR. Bonded orthodontic retainers: a review. Am J Orthod Dentofacial Orthop 1995;108:207-13.

2. Cerny R. Permanent fixed lingual retention. J Clin Orthod 2001;35:728-32.
3. Durbin DD. Relapse and the need for permanent fixed retention. J Clin Orthod 2001;35:723-7.

4. Corti AF. Technique clinic: an indirect-bonded lingual retainer. J Clin Orthod 1991;25:631-2.

5. Haydar B, Haydar S. An indirect method for bonding lingual retainers. J Clin Orthod 2001;35:608-10.

6. Becker A, Goultshin J. The multistranded retainer and splint. Am J Orthod 1984;85:470-4.

7. Bantleon HP, Droschl H. A precise and time-saving method of setting up an indirectly bonded retainer. Am J Orthod Dentofacial Orthop 1988;93:78-82.

8. Liou EJW, Chen LIJ, Huang CS. Nickel-titanium mandibular bonded lingual 3-3 retainer: for permanent retention and solving relapse of mandibular anterior crowding. Am J Orthod Dentofacial Orthop 2001;119:443-9.

9. Karaman AI, Kir N, Belli S. Four applications of reinforced polyethylene fiber material in orthodontic practice. Am J Orthod Dentofacial Orthop 2002;121:650-4.

10. Dahl EH, Zachrisson BU. Long-term experience with directbonded lingual retainers. J Clin Orthod 1991;25:619-30. 
11. Oesterle LJ, Shellhart C, Henderson S. Enhancing wire-composite bond strength of bonded retainers with wire surface treatment. Am J Orthod Dentofacial Orthop 2001;119:625-31.

12. Ormianer Z, Laufer BZ, Nissan J, Gross M. An investigation of heat transfer to the implant-bone interface related to exothermic heat generation during setting of autopolymerizing acrylic resins applied directly to an implant abutment. Int J Oral Maxillofac Implants 2000;15:837-42.
13. Cahi E, Rosen M, Becker PJ. A comparison of the dimensional stability of three inlay pattern materials. J Dent Assoc S Afr 1996;51:337-42.

14. Bearn DR, McCabe JF, Gordon PH, Aird JC. Bonded orthodontic retainers: The wire-composite interface. Am J Orthod Dentofacial Orthop 1997;111:67-74.

15. Hamula W. Direct bonding with light-cured adhesive. J Clin Orthod 1991;25:437-8 